\title{
Menaquinone-4 Accelerates Calcification of Human Aortic Valve Interstitial Cells in High-Phosphate Medium through PXR $₫$
}

\author{
Wei Yang, ${ }^{1}$ Zaiqiang Yu, ${ }^{1}$ Mari Chiyoya, Xu Liu, Kazuyuki Daitoku, Shigeru Motomura, \\ Tadaatsu Imaizumi, Ikuo Fukuda, Ken-Ichi Furukawa, Motonori Tsuji, and Kazuhiko Seya \\ Departments of Thoracic and Cardiovascular Surgery (W.Y., Z.Y., M.C., X.L., K.D., I.F.), Vascular Biology (T.I., K.S.), and \\ Pharmacology (S.M., K.-I.F.), Hirosaki University Graduate School Medicine, Hirosaki, Japan; and Institute of Molecular Function, \\ Saitama, Japan (M.T.)
}

Received October 9, 2019; accepted December 11, 2019

\begin{abstract}
Recently, we confirmed that in human aortic valve interstitial cells (HAVICs) isolated from patients with aortic valve stenosis (AVS), calcification is induced in high inorganic phosphate (highPi) medium by warfarin (WFN). Because WFN is known as a vitamin $\mathrm{K}$ antagonist, reducing the formation of blood clots by vitamin $\mathrm{K}$ cycle, we hypothesized that vitamin $\mathrm{K}$ regulates WFN-induced HAVIC calcification. Here, we sought to determine whether WFN-induced HAVIC calcification in high-Pi medium is inhibited by menaquinone-4 (MK-4), the most common form of vitamin $\mathrm{K}_{2}$ in animals. HAVICs obtained from patients with AVS were cultured in $\alpha$-modified Eagle's medium containing $10 \%$ FBS, and when the cells reached $80 \%-90 \%$ confluency, they were further cultured in the presence or absence of MK-4 and WFN for 7 days in high-Pi medium (3.2 mM Pi). Intriguingly, in high-Pi medium, MK-4 dose-dependently accelerated WFNinduced HAVIC calcification and also accelerated the calcification when used alone (at $10 \mathrm{nM}$ ). Furthermore, MK-4 enhanced alkaline phosphatase (ALP) activity in HAVICs, and 7 days of MK4 treatment markedly upregulated the gene expression of the calcification marker bone morphogenetic protein 2 (BMP2). Notably, MK-4-induced calcification was potently suppressed
\end{abstract}

by two pregnane $X$ receptor $(P X R)$ inhibitors, ketoconazole and coumestrol; conversely, PXR activity was weakly increased, but in a statistically significant and dose-dependent manner, by MK4. Lastly, in physiologic-Pi medium, MK-4 increased BMP2 gene expression and accelerated excess BMP2 $(30 \mathrm{ng} / \mathrm{ml})$-induced HAVIC calcification. These results suggest that MK-4, namely vitamin $\mathrm{K}_{2}$, accelerates calcification of HAVICs from patients with AVS like WFN via PXR-BMP2-ALP pathway.

\section{SIGNIFICANCE STATEMENT}

For aortic valve stenosis (AVS) induced by irreversible valve calcification, the most effective treatment is surgical aortic or transcatheter aortic valve replacement, but $20 \%$ of patients are deemed unsuitable because of its invasiveness. For effective drug treatment strategies for AVS, the mechanisms underlying aortic valve calcification must be elucidated. Here, we show that menaquinone- 4 accelerates warfarin-induced calcification of AVS-patient human aortic valve interstitial cells in high inorganic phosphate medium; this effect is mediated by pregnane $X$ receptor-bone morphogenetic protein 2-alkaline phosphatase signaling, which could be targeted for novel drug development.

\section{Introduction}

Aortic valve stenosis (AVS) is the most frequent adult heartvalve disease in ageing societies (Olivetti et al., 1996; Roberts and Ko, 2005); AVS prevalence is $>4.6 \%$ in people over 75 years old worldwide (Stewart et al., 1997; Nkomo et al., 2006). Calcified aortic valves are characterized by massive fibrotic thickening of valve leaflets and extensive focal ectopic calcification (Mohler, 2004). The most effective treatment of

This work was supported by JSPS KAKENHI [Grants 24590310, 16K10619, 16K10449, 17K10916, 18K16381, 19K09089] and by grants-in-aid from The Cardiovascular Research Fund, Tokyo, Japan.

${ }^{1}$ W.Y. and Z.Y. contributed equally to this work.

https://doi.org/10.1124/jpet.119.263160.

S This article has supplemental material available at jpet.aspetjournals.org.
AVS induced by irreversible valve calcification is surgical aortic valve replacement, which is extremely invasive (Iung et al., 2003), or transcatheter aortic valve replacement, which is used for high-risk patients (Hu, 2012). However, approximately $20 \%$ of patients with AVS are deemed unsuitable for valve replacement because of its invasiveness (van Geldorp et al., 2009). Thus, the development of effective medical drug-treatment strategies is essential, and this requires the elucidation of the detailed mechanism of aortic valve calcification.

For preventing and treating thromboembolic and embolic complications after surgical valve replacement or atrial fibrillation, anticoagulant therapy is necessary (Ageno et al., 2012). The most commonly prescribed postoperative anticoagulation drug is warfarin (WFN), but long-term WFN

ABBREVIATIONS: $\alpha$-MEM, $\alpha$-modified Eagle's medium; ALP, alkaline phosphatase; AVS, aortic valve stenosis; BMP2, bone morphogenetic protein 2; Ct, cycle threshold; CYP3A4, Cytochrome P450 3A4; CYP2C9, Cytochrome P450 2C9; HAVIC, human aortic valve interstitial cell; high-Pi, high inorganic phosphate; G3PDH, glyceraldehyde 3-phosphate dehydrogenase; MAP, mitogen-activated protein; MGP, matrix Gla protein; MK-4, menaquinone-4; NF- $\kappa$ B, nuclear factor kappa-B; PCR, polymerase chain reaction; PFA, phosphonoformic acid; PiT-1, Pi symporter 1; PXR, pregnane $X$ receptor; $R X R$, retinoic $X$ receptor; WFN, warfarin. 
administration has been widely shown to induce valvular calcification (Yamamoto et al., 2010; Palaniswamy et al., 2011; (Jiang et al., 2016), and hyperphosphatemia in particular has been reported to accelerate WFN-induced valvular calcification (Palaniswamy et al., 2011). Notably, our recent study demonstrated that in high inorganic phosphate (high-Pi) medium, WFN induces the ectopic calcification of human aortic valve interstitial cells (HAVICs) obtained from patients with AVS, but not patients without AVS, through the pregnane $\mathrm{X}$ receptor (PXR)-bone morphogenetic protein 2 (BMP2)-alkaline phosphatase (ALP) pathway at the cellular level (Yu et al., 2019).

WFN, a widely recognized vitamin $\mathrm{K}$ antagonist, reduces the formation of blood clots by inhibiting the vitamin $\mathrm{K}$ cycle for the biosynthesis of coagulation factors II, VII, IX, and $\mathrm{X}$ (Stafford, 2005). WFN inhibits vitamin $\mathrm{K}$ epoxide reductase, an enzyme complex responsible for the recycling of vitamin $\mathrm{K}$ in the liver (Ansell et al., 2004; Rost et al., 2004). WFN was reported to promote aortic valve calcification by inhibiting vitamin $\mathrm{K}$-dependent matrix Gla protein (MGP) (Schurgers et al., 2007), a 10-kDa secreted protein synthesized by vascular smooth muscle cells that functions through five calcium-ion-binding $\gamma$-carboxyglutamic acid (Gla) residues. Moreover, MGP was reported to inhibit vascular and valvular calcification by sequestering BMP2 and downregulating BMP signaling (Wallin et al., 2000; Yao et al., 2010). Our previous work, which demonstrated that the knockdown of MGP induces the spontaneous calcification of HAVICs, also supported the important role of BMP2 in aortic valve ectopic calcification (Chiyoya et al., 2018). Therefore, we hypothesized that vitamin $\mathrm{K}$ suppresses WFN-induced HAVIC calcification and thus could be used as a therapeutic agent for AVS.

We previously reported that WFN induces aortic valve calcification through the PXR pathway (Yu et al., 2019), and other recent studies showed that PXR is activated by WFN as well as vitamin $K_{2}$ (Tabb et al., 2003; Rulcova et al., 2010). PXR is a master regulator of xenobiotic metabolism, and elucidation of PXR activity is critical for understanding the mechanisms underlying several diseases, including inflammation (Ichikawa et al., 2006; Li et al., 2013). Furthermore, vitamin $\mathrm{K}_{2}$ can enhance collagen accumulation through PXR, resulting in bone formation (Tabb et al., 2003). However, the effect of vitamin $\mathrm{K}_{2}$ on WFN-induced calcification remains unknown.

In previous studies, we confirmed that a high Pi concentration $(3.2 \mathrm{mM})$ induces HAVIC calcification through the activation of ALP without any accompanying changes in the expression of various calcification-related genes, such as BMP2 and Runt-related transcription factor 2 (Nomura et al., 2013; Seya et al., 2016). Here, we investigated the effect of vitamin $K_{2}$ on WFN-induced calcification and the mechanism by which vitamin $\mathrm{K}_{2}$ affects aortic valve calcification. Contrary to our expectations, vitamin $\mathrm{K}_{2}$, like $\mathrm{WFN}$, was found to promote calcification of HAVICs extracted from patients with AVS by accelerating BMP2 expression and ALP activity. Furthermore, investigation of the molecular mechanism of vitamin $\mathrm{K}_{2}$-induced HAVIC calcification revealed critical roles of the PXR-BMP2 pathway in aortic valve ectopic calcification.

\section{Materials and Methods}

Materials. The following reagents were from commercial sources: $\alpha$-modified Eagle's medium ( $\alpha$-MEM) (Nacalai Tesque, Kyoto, Japan); fetal bovine serum (FBS; Biofluids) and penicillin and streptomycin (Thermo Fisher Scientific, Waltham, MA); menaquinone-4 (MK-4), WFN, and other analytical-grade reagents, including ketoconazole, coumestrol, phosphonoformic acid (PFA), and DMSO (Wako Pure Chemicals, Osaka, Japan); collagenase type V, dorsomorphin, SB239063, and U-0126 (Sigma-Aldrich, St. Louis, MO); SN-50 and BMS345541 (Cayman Chemical, Ann Arbor, MI); and Power SYBR Green PCR Master Mix (TOYOBO, Osaka, Japan). All primers used for quantitative real-time PCR were obtained from Fasmac (Kanagawa, Japan). All chemicals used were of the highest purity commercially available. All solutions were freshly prepared and were of sufficiently high concentrations to allow addition of only very small volumes to the culture medium. In all experiments, the final DMSO concentration in the culture medium was $0.1 \%$, which produced no effect on the cells or assays.

HAVIC Isolation and Culture. Human aortic valves used in the study were obtained from six patients with AVS who underwent surgical aortic valve replacement at Hirosaki University Hospital (Aomori, Japan). The mean age of the patients was $64.3 \pm 5.8$ years (three males, age $67.7 \pm 4.2$ years, and three females, age $61.0 \pm 11.1$ years). The mean serum $\mathrm{Pi}$ level of the patients immediately before aortic valve replacement was as high as $4.8 \pm 0.4 \mathrm{mg} / \mathrm{dl}$ (male: $5.4 \pm$ $0.4 \mathrm{mg} / \mathrm{dl}$; female: $4.3 \pm 0.4 \mathrm{mg} / \mathrm{dl}$ ). There was no significant difference between females and males in age or serum phosphate level. The plaque lesions and calcification exhibited similar morphology and pathology across both sexes. All patients provided written informed consent, and the study was approved by the Institutional Review Board of Hirosaki University Hospital and conformed to the principles outlined in the Declaration of Helsinki.

Human aortic valve specimens were gently cut into small pieces $(2 \pm 1 \mathrm{~mm})$ and washed with $\alpha$-MEM containing $10 \% \mathrm{FBS}$, after which HAVICs were isolated by means of treatment with collagenase type $\mathrm{V}(1 \mathrm{mg} / \mathrm{ml})$, with the minced valve specimens being incubated for 2 hours at $37^{\circ} \mathrm{C}$ in the presence of $95 \% \mathrm{O}_{2}$ and $5 \% \mathrm{CO}_{2}$. HAVICs were cultured in $\alpha$-MEM containing $10 \% \mathrm{FBS}, 100 \mathrm{U} / \mathrm{ml}$ sodium penicillin G, and $100 \mu \mathrm{g} / \mathrm{ml}$ streptomycin. After the cells reached $80 \%-90 \%$ confluency, they were further cultured in the presence or absence of MK-4 for 7 days. The medium was replenished every 3 or 4 days (Seya et al., 2016).

Identification and Evaluation of Calcification. HAVICs were seeded into 12-well plates and grown for 3 days (until 80\%-90\% confluency) and then were further cultured for 7-21 days with or without MK-4 (10 nM) and/or WFN $(1 \mu \mathrm{M})$ in medium containing phosphate $(\mathrm{Pi})$ at normal $(1 \mathrm{mM})$ or high $(3.2 \mathrm{mM})$ medium for 7 days or excess BMP2 $(30 \mathrm{ng} / \mathrm{ml})$ in normal Pi medium ( $1 \mathrm{mM}$ ) for 21 days. The degree of calcification was measured using Alizarin Red S dye; the dye-stained cells were examined under a microscope equipped with a digital camera (Canon, Tokyo, Japan), and the amount of the dye released from the extracellular matrix after incubation in $100 \mathrm{mM}$ aqueous cetylpyridinium chloride solution was spectrophotometrically quantified by measuring the 550-nm absorbance (Stanford et al., 1995).

ALP Activity Assay. HAVICs were seeded into 12-well plates and grown for 3 days (until 80\%-90\% confluency) and then were further cultured for 7-21 days in the presence or absence of MK-4 (10 nM). Proteins were collected from the cells on days 0,7 , and 21 by using a cell lysis buffer containing $80 \mu \mathrm{l} 0.05 \%$ Triton X-100, and ALP activity was measured using a LabAssay ALP Kit from Wako Pure Chemicals.

Measurement of Gene Expression. Total RNA was isolated from HAVICs by using a QuickGene RNA Cultured Cell kit S (Promega, Fitchburg, WI). An aliquot of the total RNA was reverse transcribed into cDNA by using random primers. For real-time PCR analysis, cDNA was amplified (on a CFS Real-Time System; Bio-Rad, Hercules, CA) using the following protocol: initial denaturation at $95^{\circ}$ $\mathrm{C}$ for 1 minute, followed by 40 cycles of amplification at $95^{\circ} \mathrm{C}$ for 15 seconds and $60^{\circ} \mathrm{C}$ for 1 minute. The $20-\mu \mathrm{l}$ reaction volume included $3 \mu \mathrm{l}$ 1:4 dilution of the first-strand reaction product, $0.3 \mu \mathrm{l} 10 \mu \mathrm{M}$ forward and reverse primers, $2.9 \mu \mathrm{l}$ pure water, and $5 \mu \mathrm{l} \mathrm{SYBR}$ 
quantitative PCR reagent. The primers used for ALP, BMP2, PXR, CYP3A4, CYP2C9, and glyceraldehyde 3-phosphate dehydrogenase (G3PDH) genes (Table 1) were designed using The National Center for Biotechnology Information Primer BLAST (Bethesda, MD). Amplification of the housekeeping gene $G 3 P D H$ served as a normalization standard. Real-time PCR data were represented as cycle threshold (Ct) levels and normalized by the individual G3PDH control Ct values. Relative gene expression was calculated using the $2^{-\Delta \Delta \mathrm{Ct}}$ method (Livak and Schmittgen, 2001).

Western Blotting Analysis of Phospho-Smad1/5/8. Cytoplasmic extracts were obtained by lysing cells in $20 \mathrm{mM}$ Tris-HCl, $\mathrm{pH} 7.4$, containing $0.05 \%$ Triton X-100. After measuring protein concentrations by using the Pierce Quantitative Colorimetric Peptide Assay (Thermo Fisher Scientific), $0.2 \mu \mathrm{g}$ each protein sample was separated on Bolt Bis-Tris Plus 4\%-12\% (MES) SDS-PAGE gels (ATTO Co., Tokyo, Japan), and then the proteins were transferred to PolyVinylidene DiFluoride membranes by using an iBlot Dry Blotting System (Thermo Fisher Scientific). The membranes were incubated with primary antibodies [anti-phospho-Smad1/5/8, 1:500 (Merck, Darmstadt, Germany); anti-Smad1/5/8, 1:500 (Cayman Chemical); and anti- $\beta$-actin, 1:1000 (Santa Cruz Biotechnology, Dallas, TX)] and then with Alexa Fluor 680 goat antirabbit or antimouse secondary antibody by using an iBind Western System (Thermo Fisher Scientific). Proteins were detected using an Odyssey Imaging System (LI-COR Biosciences, Lincoln, NE).

Effects of Various Drugs on MK-4-Induced Calcification of HAVICs. Cells were seeded into 12-well plates, cultured for 3 days (until 80\%-90\% confluency), and pretreated for 1 hour with inhibitors of Smad1/5/8 phosphorylation (dorsomorphin, $3 \mu \mathrm{M}$ ), nuclear factor kappa-B (NF- $\kappa \mathrm{B})$ p65 subunit translocation into the nucleus (SN-50, $10 \mu \mathrm{M})$, inhibitor of NF- $\kappa$ B kinase subunit beta (BMS345541, $10 \mu \mathrm{M}$ ), or mitogen-activated protein (MAP) kinase signaling (U-0126, $10 \mu \mathrm{M}$; SB239063, $3 \mu \mathrm{M}$ ); subsequently, the cells were treated with MK-4 (10 $\mathrm{nM}$ ) for 7 days. On day 7 , we examined alterations in calcification and ALP activity and measured gene expression by using real-time PCR.

PXR Activity Assay. IB0700 PXR (NR112), a human PXR reporter assay system $(1 \times 96$-well format assay), was obtained from Indigo Biosciences (State College, PA). PXR reporter cells were prepared using INDIGO's proprietary CryoMite process, with and without MK-4 included at various concentrations, and PXR activity was measured after culturing the cells for 24 hours.

Statistical Analysis. All statistical analyses were performed using KyPlot 5.0 software (Kyenslab, Tokyo, Japan). Group comparisons were performed by using ANOVA with the Student-NewmanKeuls post hoc correction procedure. Values are presented as means \pm S.E.M.; $P<0.05$ was considered statistically significant.

\section{Results}

Effect of MK-4 on WFN-Induced HAVIC Calcification in High-Pi Medium. To ascertain whether MK-4 inhibits the WFN effect on HAVIC calcification, HAVICs obtained

TABLE 1

Primers used for quantitative real-time PCR

\begin{tabular}{|c|c|c|}
\hline Gene & GenBank Accession No. & Sequences $\left(5^{\prime}-3^{\prime}\right)$ \\
\hline ALP & NM_000478 & $\begin{array}{l}\text { Forward: agaaccccaaaggcttcttc } \\
\text { Reverse: cttggcttttccttcatggt }\end{array}$ \\
\hline BMP2 & NM_001200 & $\begin{array}{l}\text { Forward: cggactgcggtctcctaa } \\
\text { Reverse: ggaagcagcaacgctagaag }\end{array}$ \\
\hline PXR & NM_001065 & $\begin{array}{l}\text { Forward: gcttcagaaaaccacctcagaca } \\
\text { Reverse: caataatgccggtactggttcttc }\end{array}$ \\
\hline CYP3A4 & AF182273 & $\begin{array}{l}\text { Forward: cacaaaccggaggccttttg } \\
\text { Reverse: ggtgaaggttggagacagca }\end{array}$ \\
\hline CYP2C9 & NM_000771 & $\begin{array}{l}\text { Forward: ggccatgctggttctcaaaa } \\
\text { Reverse: ctcaagtaactctaacactcacc }\end{array}$ \\
\hline G3PDH & NM_002046 & $\begin{array}{l}\text { Forward: tgcaccaccaactgcttagc } \\
\text { Reverse: ggcatggactgtggtcatgag }\end{array}$ \\
\hline
\end{tabular}

from patients with AVS were cultured for 7 days in high-Pi medium $(3.2 \mathrm{mM} \mathrm{Pi})$ in the presence or absence of MK-4 (3-100 nM). MK-4 at $>1 \mu \mathrm{M}$ triggered cell death in HAVICs. WFN induced aortic valve calcification in the high-Pi medium (Fig. 1A), and, contrary to our expectation, MK-4 potently accelerated WFN-induced HAVIC calcification (Fig. 1B). MK-4 alone did not induce HAVIC calcification, but MK-4 dose-dependently accelerated HAVIC calcification and almost reached a plateau at $10 \mathrm{nM}$ by WFN when cultured in high-Pi medium, as shown by the positive staining of the cells by Alizarin Red S (Fig. 1B). We used $10 \mathrm{nM} \mathrm{MK-4} \mathrm{in} \mathrm{our}$ subsequent experiments, which was the most suitable concentration. Spectrophotometric quantification results obtained using Alizarin Red S dye showed that MK-4 accelerated the calcification in a statistically significant manner in the presence of high $\mathrm{Pi}$ (Fig. 1C). These data suggested that HAVICs provided by patients with AVS have high sensitivity to MK-4, as they do to WFN, and thus MK-4 exposure results in the calcification of these cells in high-Pi medium.

Mechanism of MK-4-Induced HAVIC Calcification. We investigated the mechanism by which the calcification of AVS-patient HAVICs is induced by MK-4 in high-Pi medium. When HAVICs reached $80 \%-90 \%$ confluency, they were further cultured for 7 days in the presence or absence of MK$4(10 \mathrm{nM})$, and in these cells, MK-4-induced calcification in high-Pi medium was eliminated by treatment with PFA, an inhibitor of sodium-dependent Pi symporter 1 (PiT-1) (Fig. 2A).

To clarify the molecular mechanism underlying the observed effect, we measured BMP2 gene expression and ALP activity during MK-4-induced HAVIC calcification in high-Pi medium. After HAVICs were cultured with MK-4 (10 nM) in high-Pi medium for 7 days, BMP2 gene expression and ALP activity were both increased significantly (Fig. 2, B and C). In our previous study, we confirmed that in HAVICs, tumor necrosis factor- $\alpha$ stimulates BMP2 expression via the activation of transcription factor $\mathrm{NF}-\kappa \mathrm{B}$, and then the BMP2 produced induces Smad1/5/8 phosphorylation, resulting in ALP activation and then HAVIC calcification (Yu et al., 2011). Thus, we furthermore investigated the effects of the Smad1/5/ 8 phosphorylation inhibitor dorsomorphin $(3 \mu \mathrm{M})$ and NF- $\kappa \mathrm{B}$ inhibitor SN-50 (10 $\mu \mathrm{M})$ to confirm whether MK-4 induces HAVIC calcification through the NF- $\kappa$ B-BMP2-ALP pathway. We found that dorsomorphin, but not SN-50, attenuated HAVIC calcification by MK-4 in high-Pi medium (Fig. 2D). Moreover, two inhibitors of the MAP kinase pathway, U-0126 (Extracellular signal-regulated kinase inhibitor, $10 \mu \mathrm{M}$ ) and SB239063 (p38 kinase inhibitor, $3 \mu \mathrm{M}$ ), failed to inhibit the MK-4-induced calcification of HAVICs from patients with AVS (data not shown). These results indicated that MK-4 accelerates HAVIC calcification through the BMP2-ALP pathway in high-Pi medium.

Inhibitory Effect of PXR Inhibitors on HAVIC Calcification by MK-4 in High-Pi Medium. We next investigated the mechanism upstream of BMP2 expression after the stimulation of HAVICs by MK-4. Intriguingly, in HAVICs, PXR activity was increased weakly, but in a statistically significant and dose-dependent manner, by MK-4 (Fig. 3A). The average $\mathrm{EC}_{50}$ of MK-4 was $6.2 \mathrm{nM}$. To confirm that PXR produces an effect and to examine how PXR functions in MK-4-induced calcification of HAVICs obtained from patients with AVS, we used two inhibitors to alter PXR 
A

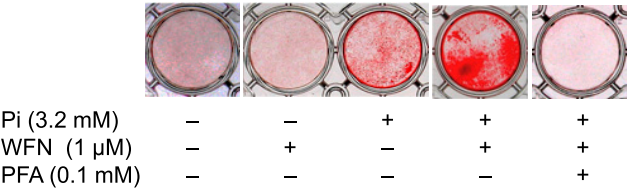

B
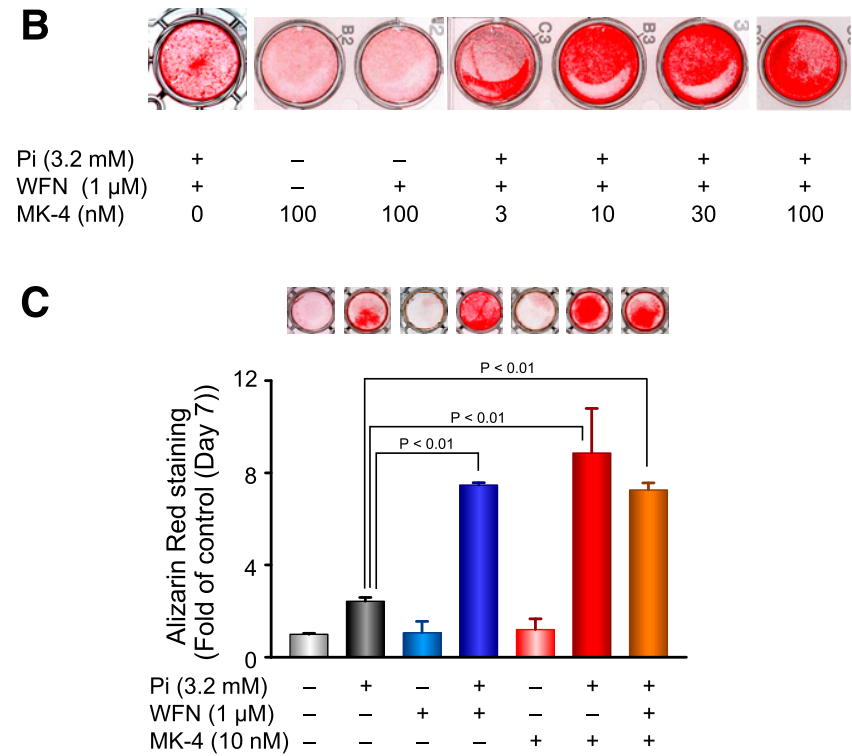

Fig. 1. Effect of MK-4 on WFN-induced HAVIC calcification in high-Pi medium. HAVICs were cultured in $\alpha$-MEM containing $10 \%$ FBS, and at $80 \%-90 \%$ confluency (day 0 ), the cells were further cultured for 7 days in high-Pi medium (3.2 mM Pi). (A) Representative images of Alizarin Red $\mathrm{S}$ staining of AVS-patient HAVICs cultured in the presence or absence of WFN $(1 \mu \mathrm{M})$. PFA $(0.1 \mathrm{mM})$, a selective inhibitor of the Pi transporter PiT1, largely inhibited HAVIC calcification induced by WFN in high-Pi medium. (B) Typical images of Alizarin Red S staining of HAVICs after WFN-induced calcification in high-Pi medium in the presence or absence of MK-4 (0-100 nM). (C) Typical images from day 7 of Alizarin Red S staining (upper images) of AVS-patient HAVICs and quantification of the Alizarin Red S staining after cetyl-pyridinium chloride extraction. The amount of released dye was quantified by measuring the absorbance at $550 \mathrm{~nm}$. All ratios were normalized to untreated control values on day 7 . White bar: untreated cells; black bar: cells treated with high Pi (3.2 mM); light-blue bar: cells treated with WFN $(1 \mu \mathrm{M})$ in normal Pi $(1.0 \mathrm{mM})$; blue bar: cells treated with WFN $(1 \mu \mathrm{M})$ in high Pi; light-red bar: cells treated with MK-4 (10 nM) in normal Pi; red bar: cells treated with MK-4 in high $\mathrm{Pi}$; orange bar: cells treated with WFN and MK-4 in high Pi. Bars represent means \pm S.E.M. $(n=5)$. One-way ANOVA with Tukey's multiple comparisons test.

activity: ketoconazole, an antagonist of the nuclear receptor PXR (Duret et al., 2006), and coumestrol, which inhibits PXR by binding to activation factor-2 region, a transcriptional coregulator (Wang et al., 2008). Either ketoconazole or coumestrol strongly suppressed HAVIC calcification induced by MK-4 in high-Pi medium (Fig. 3B); this result was supported by the quantification performed using Alizarin Red S dye (Fig. 3, C and D).

MK-4-induced BMP2 gene expression and ALP activity in HAVICs in high-Pi medium were also potently inhibited in the presence of either ketoconazole or coumestrol (Fig. 4, A and B), and Smad1/5/8 phosphorylation was also suppressed by coumestrol (Fig. 4C). These data suggested that MK-4 induces calcification of HAVICs from patients with AVS through the PXR-BMP2-ALP pathway.

BMP2 Gene Expression Induced by MK-4 in HAVICs from Patients with AVS. To confirm whether BMP2 gene expression is induced by MK-4 alone in HAVICs, we measured
$B M P 2$ expression in cells cultured in $\alpha$-MEM containing $\mathrm{Pi}$ at the physiologic concentration. In HAVICs cultured for 3 days in the presence of MK-4 (10 nM), BMP2 gene expression was notably induced, and this induction was markedly inhibited in the presence of coumestrol (Fig. 5A). We further confirmed that MK-4 treatment increased ALP activity and that this was also inhibited by coumestrol (Fig. 5B).
A

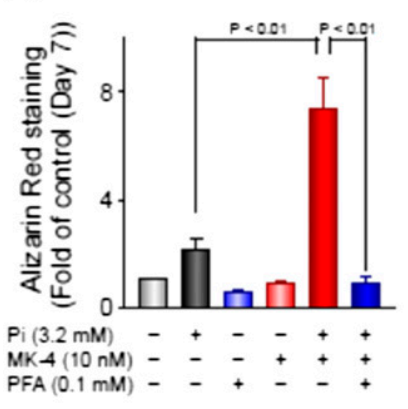

C

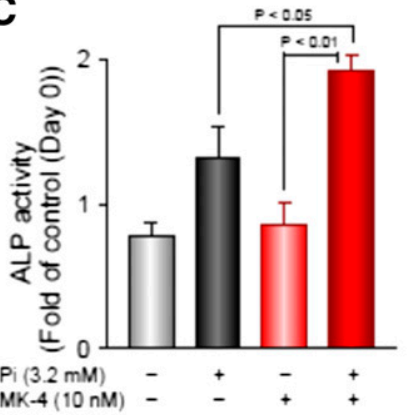

B

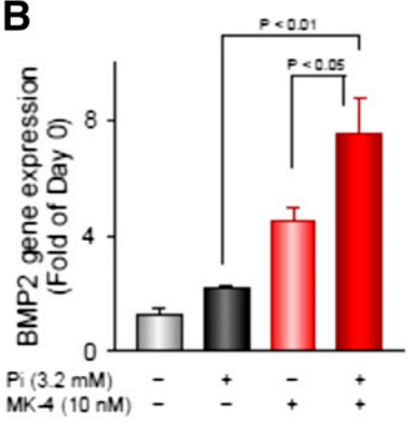

D

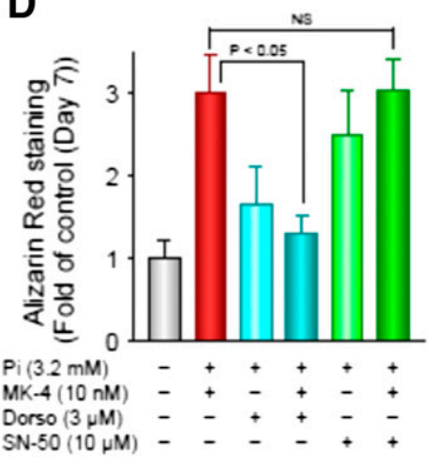

Fig. 2. AVS-patient HAVIC calcification induced by MK-4 in high-Pi medium. HAVICs from patients with AVS were cultured in $\alpha$-MEM containing $10 \% \mathrm{FBS}$, and at $80 \%-90 \%$ confluency (day 0 ), the cells were further cultured for 7 days. (A) The quantification on day 7 of Alizarin Red S staining of AVS-patient HAVICs after cetyl-pyridinium chloride extraction. The amount of released dye was quantified by measuring the 550-nm absorbance. All ratios were normalized to untreated control values on day 7 . White bar: untreated cells; black bar: cells treated with high $\mathrm{Pi}(3.2 \mathrm{mM})$; light-blue bar: cells treated with PFA $(0.1 \mathrm{mM})$ in normal $\mathrm{Pi}(1.0 \mathrm{mM})$; light-red bar: cells treated with MK-4 (10 nM) in normal Pi; red bar: cells treated with MK-4 in high Pi; blue bar: cells treated with MK-4 and PFA in high Pi. Bars represent means \pm S.E.M. $(n=5)$. Oneway ANOVA with Tukey's multiple comparisons test. (B) BMP2 gene expression and (C) ALP activity in HAVICs from patients with AVS were measured on day 7 . All ratios were calculated versus the control group on day 0 . Relative gene-expression levels were determined by normalizing measured values to those obtained for the housekeeping gene G3PDH. White bar: untreated cells; black bar: cells treated with high $\mathrm{Pi}(3.2 \mathrm{mM})$; light-red bar: cells treated with MK-4 (10 nM) in normal Pi $(1.0 \mathrm{mM})$; red bars: cells treated with MK-4 and high Pi. Bars represent means \pm S.E.M. ( $n=5$ (B), $n=4(\mathrm{C})$ ). One-way ANOVA with Tukey's multiple comparisons test. (D) HAVIC calcification induced by MK-4 in the presence or absence of Smad1/5/8 phosphorylation inhibitor dorsomorphin (Dorso) or NF-кB inhibitor SN-50 in high-Pi medium. Quantification on day 7 of Alizarin Red S staining after cetyl-pyridinium chloride extraction. The amount of released dye was quantified by measuring the $550-\mathrm{nm}$ absorbance. All ratios were normalized to the control value on day 7 . White bar: untreated cells; red bar: cells treated with $10 \mathrm{nM}$ MK-4 and high Pi (3.2 mM); lightcyan blue bar: cells treated with high $\mathrm{Pi}$ and $3 \mu \mathrm{M}$ Dorso, an inhibitor of Smad1/5/8 phosphorylation; cyan blue bar: cells treated with high Pi, MK4 , and $3 \mu \mathrm{M}$ Dorso; light-green bar: cells treated with high Pi and $10 \mu \mathrm{M}$ SN-50, an NF-кB inhibitor; green bar: cells treated with high Pi, MK-4, and SN-50. Bars represent means \pm S.E.M. $(n=5)$. One-way ANOVA with Tukey's multiple comparisons test. 
A

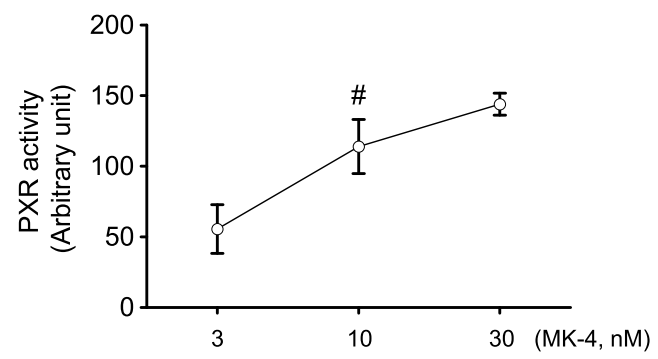

B

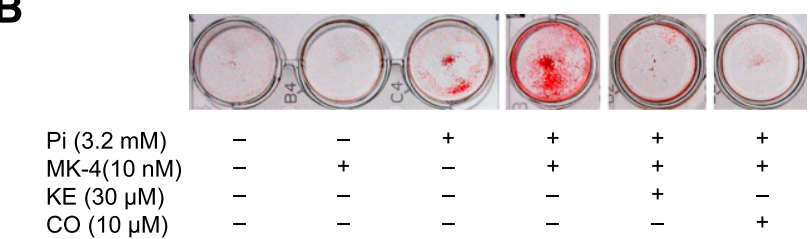

C

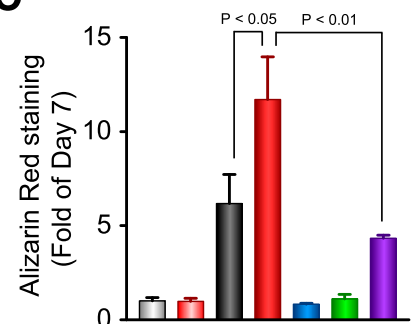

$\mathrm{Pi}(3.2 \mathrm{mM})$

MK-4 (10 nM) - $-++-\ldots+$

$\mathrm{KE}(30 \mu \mathrm{M})$

Fig. 3. AVS-patient HAVIC calcification induced by $\mathrm{MK}-4$ in high-Pi medium is suppressed by inhibitors of PXR. (A) Stimulation of PXR transcriptional activity in a mammalian PXR reporter cell line. The dosetitration of MK-4 (3-30 nM) shows that MK-4 increased PXR activity in a dose-dependent manner. The average $\mathrm{EC}_{50}$ of $\mathrm{MK}-4$ was $6.2 \mathrm{nM}$. Data represent means \pm S.E.M. $(n=3)$. One-way ANOVA with Tukey's multiple comparisons test. ${ }^{\#} P<0.05$ versus effect of $3 \mathrm{nM}$ MK-4 on PXR activity. (B-D) HAVICs from patients with AVS were cultured in $\alpha$-MEM containing $10 \% \mathrm{FBS}$, and at $80 \%-90 \%$ confluency (day 0 ), the cells were further cultured for 7 days. The panels show representative images from day 7 of Alizarin Red S staining (B) and quantification of Alizarin Red S staining after cetyl-pyridinium chloride extraction in the presence or absence of two PXR inhibitors, $30 \mu \mathrm{M}$ ketoconazole (KE) (C) or $10 \mu \mathrm{M}$ coumestrol (CO) (D). The amount of released dye was quantified by measuring the absorbance at $550 \mathrm{~nm}$. All ratios were normalized to the untreated control value on day 7. White bar: untreated cells; light-red bar: cells treated with 10 nM MK-4; black bar: cells treated with high Pi (3.2 mM); red bar: cells treated with high Pi and MK-4; light-cyan blue bar: cells treated with $30 \mu \mathrm{M} \mathrm{KE} \mathrm{(C)} \mathrm{or}$ $10 \mu \mathrm{M}$ CO (D), two PXR inhibitors; light-green bar: cells treated with KE (C) or $\mathrm{CO}(\mathrm{D})$ in high Pi; light-purple bar: cells treated with $\mathrm{KE}(\mathrm{C})$ or $\mathrm{CO}(\mathrm{D})$ in the presence of MK-4 in high Pi. Bars represent means \pm S.E.M. $(n=3)$. Oneway ANOVA with Tukey's multiple comparisons test.

To confirm the role of BMP2 in this calcification pathway, we induced HAVIC calcification by adding excess BMP2 (30 ng/ml) in the presence or absence of MK-4 in $\alpha$-MEM. Excess BMP2 induced calcification in HAVICs cultured for 21 days, MK-4 accelerated the BMP2-induced HAVIC calcification, and the effect of MK-4 was inhibited by coumestrol (10 $\mu \mathrm{M})$ (Fig. 5C). Similarly, BMP2-induced ALP activation was accelerated in the presence of MK-4, and this was again inhibited by coumestrol (Fig. 5D). These results suggest that MK-4 can stimulate BMP2 gene expression through PXR and induce HAVIC calcification in the presence of high concentrations of both $\mathrm{Pi}(3.2 \mathrm{mM})$ and BMP2 $(30 \mathrm{ng} / \mathrm{ml})$.

\section{Discussion}

Recently, we demonstrated that WFN increases valve calcification by accelerating BMP2 expression and then ALP activation through PXR in high-Pi medium (Yu et al., 2019). WFN is widely recognized to exert an anticoagulant effect by inhibiting the transformation of vitamin $\mathrm{K}_{2}$, and vitamin $\mathrm{K}_{2}$ has also been reported to activate PXR (much like WFN) and thus regulate the differentiation of osteocytes (Tabb et al., 2003). Vitamin K supplementation was also reported to promote the progression of aortic valve calcification (Brandenburg et al., 2017). Conversely, vitamin $\mathrm{K}$ intake was recently shown to prevent the acceleration of vascular calcification in

A

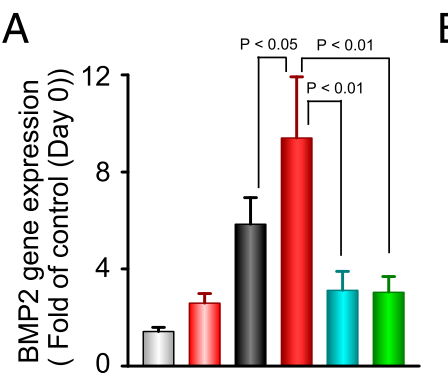

$\mathrm{B}$

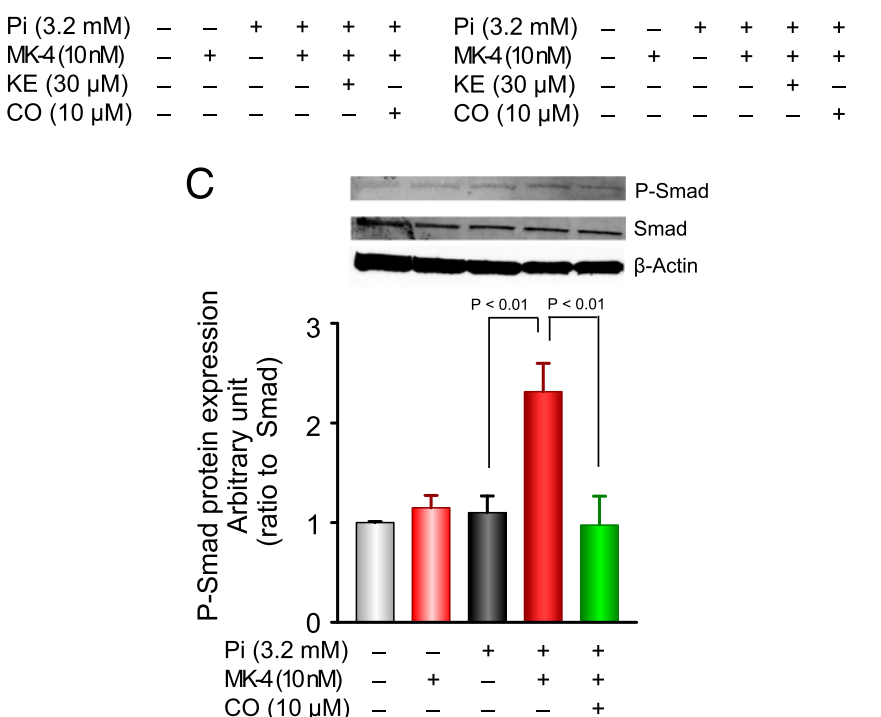

Fig. 4. MK-4-induced BMP2 expression and ALP activation in HAVICs in high-Pi medium is suppressed by PXR inhibitors. HAVICs from patients with AVS were cultured in $\alpha$-MEM containing $10 \%$ FBS, and at $80 \%-90 \%$ confluency (day 0), the cells were further cultured for 7 days. (A) $B M P 2$ gene expression and (B) ALP activity in HAVICs were measured on day 7 in the presence or absence of two PXR inhibitors, $30 \mu \mathrm{M}$ ketoconazole $(\mathrm{KE})$ or $10 \mu \mathrm{M}$ coumestrol $(\mathrm{CO})$. All ratios were calculated versus the control group on day 0 . Relative gene-expression levels were determined by normalizing measured values to those obtained for $G 3 P D H$. White bar: untreated cells; light-red bar: cells treated with 10 nM MK-4; black bar: cells treated with high Pi $(3.2 \mathrm{mM})$; red bar: cells treated with high Pi and MK-4; light-cyan blue bar: cells treated with high $\mathrm{Pi}, \mathrm{MK}-4$, and $30 \mu \mathrm{M} \mathrm{KE}$; light-green bar: cells treated with high Pi, MK-4, and $10 \mu \mathrm{M}$ CO. Bars represent means \pm S.E.M. $[n=6$ (A), $n=4$ (B)]. Oneway ANOVA with Tukey's multiple comparisons test. (C) Western blotting analysis of phospho-Smad1/5/8 (P-Smad, upper images), Smad1/5/8 (Smad, middle images), and $\beta$-actin (lower images) expression on day 1 in HAVICs isolated from patients with AVS Band intensity of P-Smad1/5/ 8 was normalized relative to Smad1/5/8 levels. Lane 1: nontreated cells; lane 2: cells treated with $10 \mathrm{nM}$ MK-4; lane 3: cells in high-Pi medium (3.2 mM Pi); lane 4: cells treated with MK-4 in high Pi; lane 5: cells treated with MK-4 in high $\mathrm{Pi}$ in the presence of $10 \mu \mathrm{M}$ CO. Values are means \pm S.E.M. $(n=3)$. One-way ANOVA with Tukey's multiple comparisons test. 
A

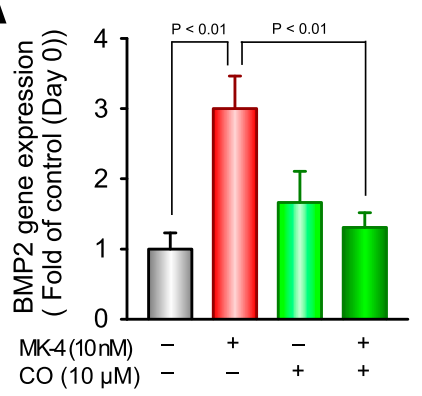

C

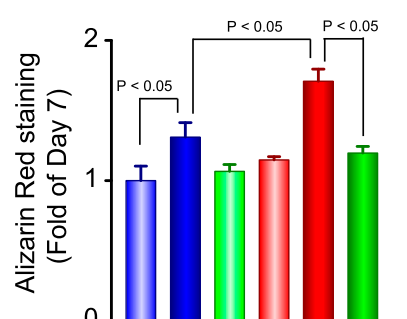

BMP2 $(30 \mu \mathrm{M})-$ $\mathrm{MK}-4(10 \mathrm{nM})$ CO $(10 \mu \mathrm{M})$

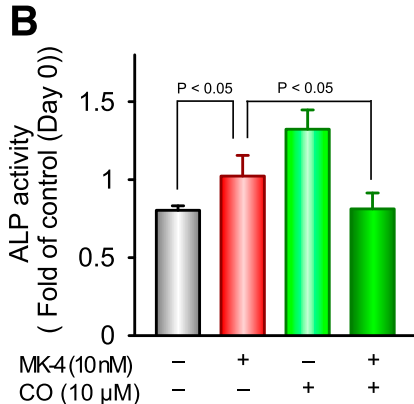

D

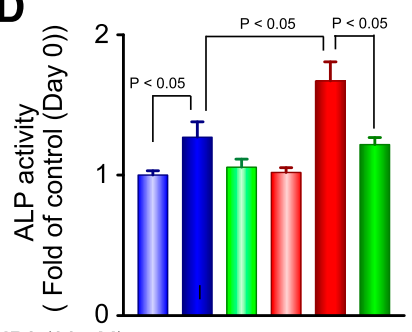

Fig. 5. MK-4 induces $B M P 2$ gene expression and activates ALP in AVSpatient HAVICs. (A) Real-time PCR analysis of BMP2 mRNA expression and (B) measurement of ALP activity in AVS-patient HAVICs following 3 days of culture in the presence or absence of MK-4/coumestrol (CO) at physiologic $\mathrm{Pi}$ concentration $(1.0 \mathrm{mM})$. All ratios were calculated versus the control group on day 0 . Relative gene-expression levels were determined by normalizing measured values to those obtained for $G 3 P D H$. White bar: untreated cells; light-red bar: cells treated with $10 \mathrm{nM}$ MK-4; light-green bar: cells treated with $10 \mu \mathrm{M}$ CO; green bar: cells treated with MK-4 in the presence of CO. Bars represent means \pm S.E.M. $[n=5(\mathrm{~A}), n=4$ (B)]. Oneway ANOVA with Tukey's multiple comparisons test. (C and D) AVS-patient HAVIC calcification induced by excess BMP2 $(30 \mathrm{ng} / \mathrm{ml})$ in the presence or absence of MK-4 $(10 \mathrm{nM})$ or CO $(10 \mu \mathrm{M})$. Quantification on day 21 of $(\mathrm{C})$ Alizarin Red S staining after cetyl-pyridinium chloride extraction and (D) ALP activity. The amount of released dye was quantified by measuring the absorbance at $550 \mathrm{~nm}$. All ratios were normalized to the untreated control value on day 7. Light-blue bar: untreated cells; blue bar: cells treated with $30 \mathrm{ng} / \mathrm{ml}$ BMP2; light-green bar: cells treated with $10 \mu \mathrm{M} \mathrm{CO}$; light-red bar: cells treated with $10 \mathrm{nM}$ MK-4; red bar: cells treated with BMP2 in the presence of MK-4; green bar: cells treated with BMP2 in the presence of MK-4 and CO. Bars represent means \pm S.E.M. $[n=6$ (C), $n=5$ (D)] One-way ANOVA with Tukey's multiple comparisons test.

patients receiving WFN treatment (Caluwé et al., 2016). Thus, the role of vitamin $\mathrm{K}_{2}$ in vascular and valvular calcification remains debated. In this study, we sought to clarify the effect of vitamin $\mathrm{K}_{2}$, namely MK-4, on WFN-induced HAVIC calcification in high-Pi medium.

Interestingly, we found here that MK-4 augmented WFNinduced calcification and further MK-4 alone promoted HAVIC calcification in high-Pi medium. Furthermore, $B M P 2$ gene expression, Smad1/5/8 phosphorylation, and ALP activity were markedly upregulated in high-Pi medium together with the activation of PXR. We further confirmed that PFA (inhibitor of PiT-1) and dorsomorphin (inhibitor of Smad1/5/8), but not SB239063 (inhibitor of MAP kinase) or SN-50 (inhibitor of NF- $\kappa$ B), suppressed calcification and ALP activation induced by MK-4 in high-Pi medium. We previously confirmed that PFA inhibits high-Pi-induced HAVIC calcification (Yu et al., 2019). BMP2 is known to stimulate the transcription factors Smad1/5/8 and then activate ALP through the expression of calcification-related genes (Yu et al., 2011). In our previous study, the most abundantly expressed calcification-related gene was distal-less homeobox 5 (Yu et al., 2011). From these results, we conclude that MK-4, like WFN, induces HAVIC calcification in high-Pi medium through the PXR-BMP2-ALP pathway. We previously showed that SR12813, a selective agonist of PXR, also accelerated HAVIC calcification in high$\mathrm{Pi}$ medium together with the enhancement of $B M P 2$ gene expression and ALP activity (Yu et al., 2019). However, the relationship between PXR activation and BMP2 expression remains unclear.

To ascertain whether PXR activation by MK-4 induces BMP2 expression, we pharmacologically investigated the MK-4 effect in normal-Pi medium. Inclusion of MK-4 alone in 3 days culture in normal-Pi medium weakly but significantly induced BMP2 expression and ALP activation, and both effects were strongly suppressed in the presence of the PXR inhibitor coumestrol. In normal-Pi medium, although MK-4 alone cannot induce the HAVIC calcification, MK-4 significantly promoted excess BMP2 (30 ng/ml)-induced HAVIC calcification, which was potently inhibited by coumestrol. These results indicate that in normal-Pi medium, MK-4 creates a calciotropic condition by BMP2 expression in HAVICs via PXR activation. We propose that the acceleration of ALP activation by MK-4 through the PXR-BMP2 pathway and the increase in Pi intake through PiT-1 additively and/or synergistically induce HAVIC calcification (Fig. 6).

PXR, a key regulator of the detoxification of xeno- and endobiotics, activates various cytochrome P450 enzymes, particularly CYP3A4 (Hakkola et al., 2018). We confirmed here that MK-4 did not alter PXR gene expression, but in highPi medium, MK-4 accelerated the expression of CYP3A4, a main detoxification enzyme and a target gene of PXR, but not CYP2C9 (Supplemental Fig. 1). CYP2C9 is recognized as the enzyme responsible for the metabolic clearance of WFN (Sconce et al., 2005). These findings support our result showing that MK-4 accelerates WFN-induced calcification of HAVICs isolated from patients with AVS (Fig. 1B). Furthermore, this increment of CYP3A4 expression in high-Pi medium suggests one possibility that activated PXR induces $B M P 2$ expression, resulting in HAVICs calcification. However, both WFN and SR12813 strongly downregulated CYP3A4 gene expression (Yu et al., 2019). Evangelista et al. (2013) have reported that in cardiac tissue, CYP3A4 is rarely expressed. One possibility suggested by these results is that MK-4 and WFN regulate the promoter activity of CYP3A4 through distinct mechanisms in HAVICs. In future work, we will investigate the effects of the aforementioned compounds on PXR-induced CYP3A4 gene expression in HAVICs by using hepatocytes as a positive control, with the aim being to elucidate the detailed molecular mechanism of HAVIC calcification through PXR in the aortic valve.

Vitamin $\mathrm{K}_{2}$ and WFN exhibit structural similarity, featuring a naphthoquinone skeleton and a coumarin skeleton, respectively. WFN exerts an anticoagulant effect and prevents thrombus formation by irreversibly and strongly inhibiting the activities of two enzymes in the vitamin $\mathrm{K}$ metabolic cycle: vitamin $\mathrm{K}$-dependent epoxide reductase and vitamin $\mathrm{K}$ quinone reductase. Intriguingly, in docking analysis (Tsuji et al., 2015), both MK-4 and WFN were found to bind to PXR at the same site. We hypothesize that the conformational change induced by the binding of WFN, MK-4, and SR12813 to PXR promotes BMP2 expression, which results in the ectopic calcification of the aortic valve (Supplemental Fig. 2). 


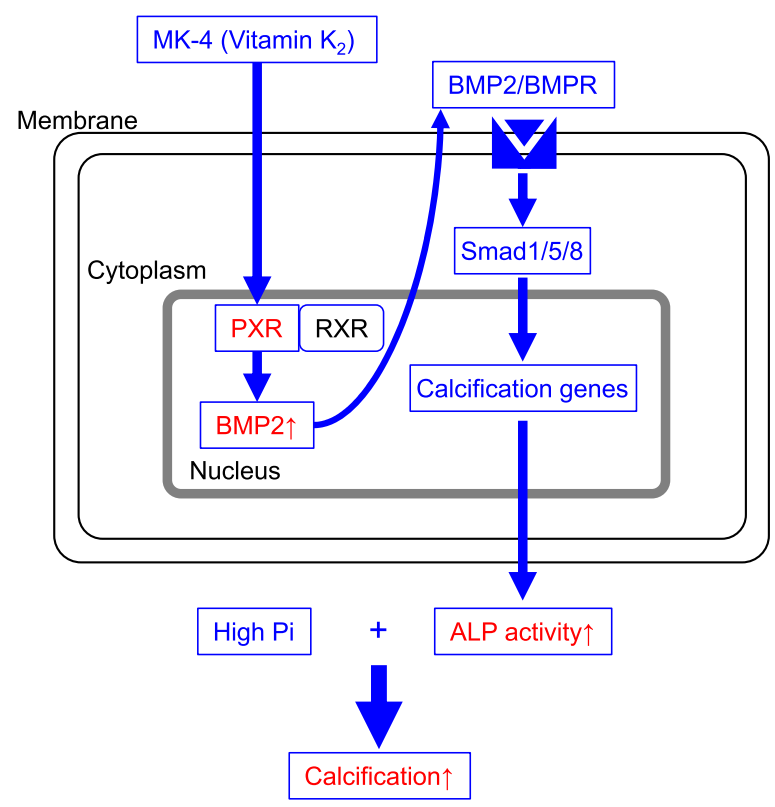

Fig. 6. Proposed mechanism of AVS-patient HAVIC calcification induced by MK-4. Arrows: pathways supported by the data obtained in this study. MK-4 accelerates BMP2 gene expression through PXR or PXR/RXR heterodimer; the produced BMP2 activates BMP receptor (BMPR), which, in turn, stimulates the expression of calcification-related genes such as distal-less homeobox 5 and Runt-related transcription factor 2 through Smad1/ $5 / 8$ in an autocrine and/or paracrine manner; this results in ALP activation (Holleville et al., 2007; Yu et al., 2011). Ultimately, ALP activation by MK-4 through the PXR-BMP2 pathway and the increase in Pi intake through PiT-1 additively and/or synergistically induce HAVIC calcification.

We have three limitations in this study. First, we could not demonstrate here how PXR activation stimulates BMP2 expression. PXR has been reported to upregulate BMP2 expression in rat hepatic cells (Roques et al., 2013). PXR is known to exert its transcriptional regulatory functions by forming a heterodimer with retinoic X receptor (RXR). RXR also has been reported to upregulate BMP2 expression in mice (Nallamshetty et al., 2013). In the future, we will investigate the detailed molecular mechanism of PXR- or PXR/RXRinduced BMP2 expression. Second, we have not demonstrated the role of the identified pathway through in vivo investigation. Various inhibitors of this pathway could represent candidate drugs for blocking aortic valve ectopic calcification. Thus, in upcoming studies, we will investigate whether rat aortic valve calcification induced by high-Pi or high BMP expression is accelerated in the presence of WFN, MK-4, and/or PXR activator such as SR12813 and then investigate the effects of various PXR, BMP2, and/or ALP inhibitors as therapeutic drugs. Third, we used HAVICs but not mesenchymal undifferentiated cells for our investigations. We recently observed in calcified aortic valves that mesenchymal undifferentiated cells were abundant; expressed the surface markers CD73, CD90, and CD105; lacked CD34 and CD45 expression; and showed enhanced sensitivity to the induction of calcification (Nomura et al., 2013). Currently, we are investigating the separation conditions that are appropriate for isolating these undifferentiated cells from HAVICs while also maintaining high viability of the undifferentiated cells. In the future, these undifferentiated cell populations in HAVICs should be investigated to determine the physiologic and pathophysiological roles of WFN and MK-4.
In summary, we demonstrated that vitamin $\mathrm{K}_{2}$, i.e., MK-4, increased PXR activity dose dependently to accelerate aortic valve calcification by elevating BMP2 expression and ALP activity in high-Pi condition. In docking analysis, we first demonstrated that not only MK-4 but also WFN can bind to PXR in the same site. These results should help clarify and propose a new mechanism of drug-induced aortic valve calcification in hyperphosphatemia. Clinically, many chronic kidney disease patients develop hyperphosphatemia, which leads to AVS by continuous hemodialysis. Our data suggests a possibility for the development of a new prevention method of AVS in hyperphosphatemia targeting PXR.

\section{Acknowledgments}

We are grateful to Yasuyuki Ishibashi of Hirosaki University for his consistent help, support, and encouragement.

\section{Authorship Contributions}

Participated in research design: Yu, Motomura, Furukawa, Seya. Conducted experiments: Yang, Yu, Chiyoya, Liu, Furukawa, Seya. Contributed new reagents or analytic tools: Yu, Daitoku, Motomura, Fukuda, Furukawa, Seya, Tsuji, Imaizumi.

Performed data analysis: Yang, Yu, Chiyoya, Liu, Furukawa, Seya. Wrote or contributed to the writing of the manuscript: Yang, Yu, Daitoku, Fukuda, Furukawa, Seya, Tsuji, Imaizumi.

Note Added in Proof-It was discovered the wrong rightmost image in Figure 1A was published in the Fast Forward version that appeared online December 16, 2019. Figure 1A has now been corrected.

\section{References}

Ageno W, Gallus AS, Wittkowsky A, Crowther M, Hylek EM, and Palareti G (2012) Oral anticoagulant therapy: antithrombotic therapy and prevention of thrombosis, 9th ed: american college of chest physicians evidence-based clinical practice guidelines, Chest 141, p e44S-e88S.

Ansell J, Hirsh J, Poller L, Bussey H, Jacobson A, and Hylek E (2004) The pharmacology and management of the vitamin K antagonists: the Seventh ACCP Conference on Antithrombotic and Thrombolytic Therapy [published correction appears in Chest (2005) 127:415-416]. Chest 126 (3 Suppl):204S-233S.

Brandenburg VM, Reinartz S, Kaesler N, Krüger T, Dirrichs T, Kramann R, Peeters F, Floege J, Keszei A, Marx N, et al. (2017) Slower progress of aortic valve calcification with vitamin $\mathrm{K}$ supplementation: results from a prospective interventional proof-of-concept study. Circulation 135:2081-2083.

Caluwé R, Pyfferoen L, De Boeck K, and De Vriese AS (2016) The effects of vitamin K supplementation and vitamin $\mathrm{K}$ antagonists on progression of vascular calcification: ongoing randomized controlled trials. Clin Kidney J 9:273-279.

Chiyoya M, Seya K, Yu Z, Daitoku K, Motomura S, Imaizumi T, Fukuda I, and Furukawa K-I (2018) Matrix Gla protein negatively regulates calcification of human aortic valve interstitial cells isolated from calcified aortic valves. J Pharmacol Sci 136:257-265.

Duret C, Daujat-Chavanieu M, Pascussi JM, Pichard-Garcia L, Balaguer P, Fabre JM, Vilarem MJ, Maurel P, and Gerbal-Chaloin S (2006) Ketoconazole and miconazole are antagonists of the human glucocorticoid receptor: consequences on the expression and function of the constitutive androstane receptor and the pregnane $\mathrm{X}$ receptor. Mol Pharmacol 70:329-339.

Evangelista EA, Kaspera R, Mokadam NA, Jones JP III, and Totah RA (2013) Activity, inhibition, and induction of cytochrome P450 2J2 in adult human primary cardiomyocytes. Drug Metab Dispos 41:2087-2094.

Hakkola J, Bernasconi C, Coecke S, Richert L, Andersson TB, and Pelkonen O (2018) Cytochrome $\mathrm{P} 450$ induction and xeno-sensing receptors pregnane $\mathrm{X}$ receptor, constitutive androstane receptor, aryl hydrocarbon receptor and peroxisome proliferator-activated receptor $\alpha$ at the crossroads of toxicokinetics and toxicodynamics. Basic Clin Pharmacol Toxicol 123 (Suppl 5):42-50.

Holleville N, Matéos S, Bontoux M, Bollerot K, and Monsoro-Burq AH (2007) Dlx5 drives Runx2 expression and osteogenic differentiation in developing cranial suture mesenchyme. Dev Biol 304:860-874.

Hu PP (2012) TAVR and SAVR: current treatment of aortic stenosis. Clin Med Insights Cardiol 6:125-139.

Ichikawa T, Horie-Inoue K, Ikeda K, Blumberg B, and Inoue S (2006) Steroid and xenobiotic receptor SXR mediates vitamin $\mathrm{K}_{2}$-activated transcription of extracellular matrix-related genes and collagen accumulation in osteoblastic cells. J Biol Chem 281:16927-16934.

Iung B, Baron G, Butchart EG, Delahaye F, Gohlke-Bärwolf C, Levang OW, Tornos P, Vanoverschelde JL, Vermeer F, Boersma E, et al. (2003) A prospective survey of patients with valvular heart disease in Europe: the Euro Heart Survey on Valvular Heart Disease. Eur Heart J 24:1231-1243. 
Jiang X, Tao H, Qiu C, Ma X, Li S, Guo X, Lv A, and Li H (2016) Vitamin $\mathrm{K}_{2}$ regression aortic calcification induced by warfarin via Gas6/Axl survival pathway in rats. Eur $J$ Pharmacol 786:10-18.

Li H, Redinbo MR, Venkatesh M, Ekins S, Chaudhry A, Bloch N, Negassa A, Mukherjee P, Kalpana G, and Mani S (2013) Novel yeast-based strategy unveils antagonist binding regions on the nuclear xenobiotic receptor PXR. J Biol Chem 288:13655-13668.

Livak KJ and Schmittgen TD (2001) Analysis of relative gene expression data using realtime quantitative PCR and the 2(-Delta Delta C(T)) method. Methods 25:402-408.

Mohler ER III (2004) Mechanisms of aortic valve calcification. Am J Cardiol 94: 1396-1402, A6.

Nallamshetty S, Wang H, Rhee EJ, Kiefer FW, Brown JD, Lotinun S, Le P, Baron R, Rosen CJ, and Plutzky J (2013) Deficiency of retinaldehyde dehydrogenase 1 induces BMP2 and increases bone mass in vivo. PLoS One 8:e71307.

Nkomo VT, Gardin JM, Skelton TN, Gottdiener JS, Scott CG, and Enriquez-Sarano M (2006) Burden of valvular heart diseases: a population-based study. Lancet 368 $1005-1011$.

Nomura A, Seya K, Yu Z, Daitoku K, Motomura S, Murakami M, Fukuda I, and Furukawa K (2013) CD34-negative mesenchymal stem-like cells may act as the cellular origin of human aortic valve calcification. Biochem Biophys Res Commun 440:780-785.

Olivetti G, Quaini F, Sala R, Lagrasta C, Corradi D, Bonacina E, Gambert SR, Cigola $\mathrm{E}$, and Anversa $\mathrm{P}$ (1996) Acute myocardial infarction in humans is associated with activation of programmed myocyte cell death in the surviving portion of the heart. $J$ Mol Cell Cardiol 28:2005-2016.

Palaniswamy C, Sekhri A, Aronow WS, Kalra A, and Peterson SJ (2011) Association of warfarin use with valvular and vascular calcification: a review. Clin Cardiol $\mathbf{3 4}$ $74-81$.

Roberts WC and Ko JM (2005) Frequency by decades of unicuspid, bicuspid, and tricuspid aortic valves in adults having isolated aortic valve replacement for aortic stenosis, with or without associated aortic regurgitation. Circulation 111:920-925.

Roques BB, Leghait J, Lacroix MZ, Lasserre F, Pineau T, Viguié C, and Martin PG (2013) The nuclear receptors pregnane $\mathrm{X}$ receptor and constitutive androstane receptor contribute to the impact of fipronil on hepatic gene expression linked to thyroid hormone metabolism. Biochem Pharmacol 86:997-1039.

Rost S, Fregin A, Ivaskevicius V, Conzelmann E, Hörtnagel K, Pelz HJ, Lappegard K Seifried E, Scharrer I, Tuddenham EG, et al. (2004) Mutations in VKORC1 cause warfarin resistance and multiple coagulation factor deficiency type 2. Nature 427: 537-541.

Rulcova A, Prokopova I, Krausova L, Bitman M, Vrzal R, Dvorak Z, Blahos J, and Pavek P (2010) Stereoselective interactions of warfarin enantiomers with the pregnane $\mathrm{X}$ nuclear receptor in gene regulation of major drug-metabolizing cytochrome P450 enzymes. J Thromb Haemost 8:2708-2717.

Schurgers LJ, Spronk HMH, Skepper JN, Hackeng TM, Shanahan CM, Vermeer C, Weissberg PL, and Proudfoot D (2007) Post-translational modifications regulate matrix Gla protein function: importance for inhibition of vascular smooth muscle cell calcification. J Thromb Haemost 5:2503-2511.

Sconce EA, Khan TI, Wynne HA, Avery P, Monkhouse L, King BP, Wood P, Kesteven $\mathrm{P}$, Daly AK, and Kamali F (2005) The impact of CYP2C9 and VKORC1 genetic polymorphism and patient characteristics upon warfarin dose requirements: proposal for a new dosing regimen. Blood 106:2329-2333.
Seya K, Furukawa K, Chiyoya M, Yu Z, Kikuchi H, Daitoku K, Motomura S, Murakam M, Oshima Y, and Fukuda I (2016) 1-Methyl-2-undecyl-4(1H)-quinolone, a derivative of quinolone alkaloid evocarpine, attenuates high phosphate-induced calcification of human aortic valve interstitial cells by inhibiting phosphate cotransporter PiT-1. J Pharmacol Sci 131:51-57.

Stanford CM, Jacobson PA, Eanes ED, Lembke LA, and Midura RJ (1995) Rapidly forming apatitic mineral in an osteoblastic cell line (UMR 106-01 BSP). J Biol Chem 270:9420-9428.

Stafford DW (2005) The vitamin K cycle. J Thromb Haemost 3:1873-1878.

Stewart BF, Siscovick D, Lind BK, Gardin JM, Gottdiener JS, Smith VE, Kitzman DW, and Otto CM (1997) Clinical factors associated with calcific aortic valve disease. Cardiovascular Health Study. J Am Coll Cardiol 29:630-634.

Tabb MM, Sun A, Zhou C, Grün F, Errandi J, Romero K, Pham H, Inoue S, Mallick S, Lin $M$, et al. (2003) Vitamin $\mathrm{K}_{2}$ regulation of bone homeostasis is mediated by the steroid and xenobiotic receptor SXR. J Biol Chem 278:43919-43927.

Tsuji M, Shudo K, and Kagechika H (2015) Docking simulations suggest that alltrans retinoic acid could bind to retinoid X receptors. J Comput Aided Mol Des 29: 975-988.

van Geldorp MW, van Gameren M, Kappetein AP, Arabkhani B, de Groot-de Laat LE, Takkenberg JJ, and Bogers AJ (2009) Therapeutic decisions for patients with symptomatic severe aortic stenosis: room for improvement? Eur $J$ Cardiothorac Surg 35:953-957, discussion 957.

Wallin R, Cain D, Hutson SM, Sane DC, and Loeser R (2000) Modulation of the binding of matrix Gla protein (MGP) to bone morphogenetic protein-2 (BMP-2). Thromb Haemost 84:1039-1044.

Wang H, Li H, Moore LB, Johnson MD, Maglich JM, Goodwin B, Ittoop OR, Wisely B, Creech K, Parks DJ, et al. (2008) The phytoestrogen coumestrol is a naturally occurring antagonist of the human pregnane X receptor. Mol Endocrinol 22: 838-857.

Yamamoto K, Yamamoto H, Yoshida K, Kisanuki A, Hirano Y, Ohte N, Akasaka T, Takeuchi M, Nakatani S, Ohtani T, et al. (2010) Prognostic factors for progression of early- and late-stage calcific aortic valve disease in Japanese: the Japanese Aortic Stenosis Study (JASS) Retrospective Analysis. Hypertens Res 33:269-274.

Yao Y, Bennett BJ, Wang X, Rosenfeld ME, Giachelli C, Lusis AJ, and Boström KI (2010) Inhibition of bone morphogenetic proteins protects against atherosclerosis and vascular calcification. Circ Res 107:485-494.

Yu Z, Seya K, Chiyoya M, Daitoku K, Motomura S, Imaizumi T, Fukuda I, and Furukawa K-I (2019) Warfarin calcifies human aortic valve interstitial cells at high-phosphate conditions via pregnane X receptor. J Bone Miner Metab 37: 944-956.

Yu Z, Seya K, Daitoku K, Motomura S, Fukuda I, and Furukawa K (2011) Tumor necrosis factor- $\alpha$ accelerates the calcification of human aortic valve interstitial cells obtained from patients with calcific aortic valve stenosis via the BMP2-Dlx5 pathway. J Pharmacol Exp Ther 337:16-23.

Address correspondence to: Dr. Kazuhiko Seya, Department of Vascular Biology, Hirosaki University Graduate School of Medicine, 5 Zaifu-cho, Hirosaki 036-8562, Japan. E-mail: seya@hirosaki-u.ac.jp 